“ (C) 2017 IEEE. Personal use of this material is permitted. Permission from IEEE must be obtained for all other uses, in any current or future media, including

reprinting/republishing this material for advertising or promotional purposes, creating new collective works, for resale or redistribution to servers or lists, or reuse of any copyrighted component of this work in other works." 


\title{
Improved Signal Interpretation for Cast Iron Thickness Assessment based on Pulsed Eddy Current Sensing
}

\author{
Linh Nguyen, Nalika Ulapane, Jaime Valls Miro, Gamini Dissanayake and Fredy Munoz \\ Centre for Autonomous Systems \\ Faculty of Engineering and Information Technology \\ University of Technology Sydney, Australia \\ Email: \{vanlinh.nguyen, nalika.ulapane, jaime.vallsmiro, gamini.dissanayake, fredy.munoz\}@uts.edu.au
}

\begin{abstract}
This paper presents a novel signal processing approach for computing thickness of ferromagnetic cast iron material, widely employed in older infrastructure such as water mains or bridges. Measurements are gathered from a Pulsed Eddy Current (PEC) based sensor placed on top of the material, with unknown lift-off, as commonly used during non-destructive testing (NDT). The approach takes advantage of an analytical logarithmic model proposed in the literature for the decaying voltage induced at the PEC sensor pick-up coil. An increasingly more accurate and robust algorithm is proven here by means of an Adaptive Least Square Fitting Line (ALSFL) recursive strategy, suitable to recognize the most linear part of the sensor's logarithmic output voltage for subsequent gradient computation, from which thickness is then derived. Moreover, efficiency is also gained as processing can be carried out on only one decaying voltage signal, unlike averaging over multiple measurements as is usually done in the literature. Importantly, the new signal processing methodology demonstrates highest accuracies at the lower thicknesses, a circumstance most relevant to NDT evaluation. Experiments that verify the proposed method in real-world thickness assessment of cast iron material are presented and compared with current practices, showing promising results.
\end{abstract}

\section{MOTIVATION AND BACKGROUND}

$\mathrm{C}$ AST iron has been extensively utilized in fabrication of vessels and pipes in industries such as transportation and distribution of power generation, chemical, oil, gas and especially water. In Australia, older critical water pipes are primarily made of cast iron material [1]. As these buried assets may have been laid over 100 years ago, accurate knowledge and evaluating of the remaining life of the pipeline network is paramount for the efficient management of the asset infrastructure by water utility companies: better water mains renewal program, breaks reduction, customer staiusfaction and minimization of replacement costs [2]. An example of a $600 \mathrm{~mm}$ diameter cast iron pipe thickness section of a water main excavated in Sydney is shown in Fig. 1, where a critical patch of low remaining wall thickness is clearly visible. Assessing cast iron thickness in those infrastructures can be conducted by in-service testing techniques, also refered to as NonDestructive Testing/ Evaluation, or NDT/NDE. These methods

This work was supported by the Critical Pipes Project funded by Sydney Water Cooperation and University of Technology Sydney, Australia.

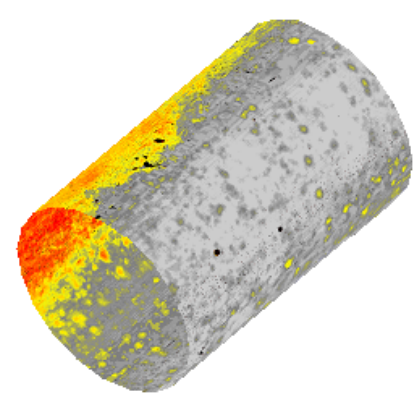

(a)

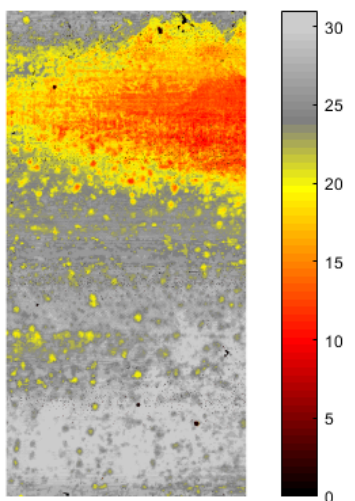

(b)
Fig. 1: Water pipe thickness interpretations (mm) in (a) 3D and (b) 2D.

comprise mostly techniques related to magnetic flux leakage (MFL), remote field Eddy current (RFEC), Eddy current (EC), pulsed Eddy current (PEC) or acoustics [3]. Whilst solutions encompassing these techniques have been commercialised by various providers around the world, accurate interpretations of the signals acquired remains challenging. For example, MFL is less sensitive to deep interacting defects, whilst remnant magnetization in the material after evaluation could be an issue. RFEC generally requires bulky geometric configuration as well as expensive power arrangements. EC - which is based on single-frequency sinusoidal signals - can only detect a specific material thickness. PEC is widely employed for NDE with ferromagnetic and non-ferromagnetic materials [4]-[7]. By using a broad-band frequency excitation via a pulse, the PEC method has multiple advantages. For instance, due to the multi-frequency pulse signal, noise on the output signal can be suppressed by the different sources [8]. Furthermore, larger frequency components allow the sensing system to detect a wider range of material thicknesses, which means a greater depth of penetration. Other advantages include simple operation, high testing speed, less expensive structure and 
robustness against interferences. On the other hand, PEC is also renowned as a non-contacting technology, applicable for evaluating the remaining material of a given infrastructure (e.g. a pipe) where the material may have been insulated by a protected layer, or simply by the presence of corrosion by-products on the pipe wall, hence inducing sensor lift-off. This effect is also apparent in drinking water mains when inspected from the inside, as they are often lined by an internal cementitious layer. A PEC sensor architecture consists of an exciter coil and a magnetic receiver, Ulapane [9] demonstrated that a non-receiver coil based design using magnetic sensors such as Hall-effect and magnetoresistive sensors has limited applicability; hence, in this work, a solenoid coil was employed as pick-up coil in the PEC sensor. Generally speaking, due to the nonlinear and heterogeneous ferromagnetic nature of the cast iron, the decaying voltage curves induced by the pick-up coil in the PEC sensor are usually accompanied by noise. In order to denoise the signals, the authors in [5], [10] proposed an average filter that computes an averaged decaying voltage signal from multiple measurements before estimating the thickness of the material. Nevertheless, requiring multiple sensor readings for calculating one thickness in this method is not feasible in realistic applications where testing speed is also a crucial factor. Therefore, in this paper we propose a new approach to interpret the PEC sensor's output signal as the thickness of the cast iron underneath the PEC sensor footprint. The main improvement in the proposed method as compared with the literature is that it only requires one sensor measurement, e.g. one decaying voltage curve, to estimate one thickness of the material. More particularly, the proposed algorithm plays interpretation better at low thicknesses and runs much faster than the conventional methods. The approach implemented in the real-life experiments using the cast iron calibration plates has shown promising results. The remaining of the paper is structured as follows. Section II introduces the principle of the PEC, which is then employed to drive an analytical solution of interpreting the cast iron thickness based on a gradient of an adaptive least square fitting line (ALSFL) of logarithmic decaying curves. A proposed algorithm of only processing one decaying voltage curve measurement collected by the PEC sensor being able to estimate the material thickness is presented in Section III. Section IV delineates the experimental implementation and results before conclusions are summarized in Section V.

\section{Pulsed Eddy Current Based Approach}

An inspection technique based on the PEC technology is introduced in this section, where the material thickness interpretation is analytically derived.

\section{A. Principle of PEC Inspection}

The PEC technique is the NDE sensor being excited by a voltage or a current pulse, which has been proved to be significantly versatile over the other EC methods and therefore is used for condition assessment of a wide variety of materials [11]. In this technique, rising and falling edges of the pulsed excitation can be theoretically described by a Heaviside step function. The Fourier transform of the Heaviside function is known to be $\delta(f)+\frac{1}{i 2 \pi f}$ where $i=\sqrt{-1}, f$ denotes frequency and $\delta(f)$ denotes the unit impulse function of $f$. This result clearly suggests that the power of low frequencies can be very high. A power of that magnitude may not be achievable by exciting with a single low frequency due to the limitations of excitation circuitry. However, a pulse enables having such desired high powers in the low frequency range while enabling a wide frequency spectrum to be contained within the magnetic field. The PEC technique can therefore achieve admirable penetration capability.

\section{B. Material Thickness Interpretation}

In circuit theory, the pick-up coil based PEC sensor is supposed to be modelled by infinitely many mutually coupled coils [6]. Therefore, by ignoring the oscillations, the decaying part of a PEC induced detector coil voltage can be formulated by

$$
V(t)=\sum_{i=1}^{\infty} b_{i} \exp \left(-c_{i} t\right)
$$

where $b_{i}$ and $c_{i}$ are constants which contain the properties of the sensor setup and the test piece. The condition $c_{i}>0$ holds for all $i$ [6]. By means of linear and homogeneous representation of magnetic permeability $\mu$ and electrical conductivity $\sigma$, the diffusion time constant of eddy currents induced in a ferromagnetic plate of thickness $d$ is defined as $\mu \sigma d^{2} / \pi^{2}$ [12]. Hence, $V(t)$ is rewritten as

$$
V(t)=b_{1} \exp \left(\frac{-\pi^{2} t}{\mu \sigma d^{2}}\right)+\sum_{i=2}^{\infty} b_{i} \exp \left(-c_{i} t\right)
$$

If $V(t)$ is differentiated in natural logarithmic form, it yields

$$
\frac{\mathrm{d} \ln [V(t)]}{\mathrm{d} t}=-\frac{\frac{b_{1} \pi^{2}}{\mu \sigma d^{2}} \exp \left(\frac{-\pi^{2} t}{\mu \sigma d^{2}}\right)+\sum_{i=2}^{\infty} b_{i} c_{i} \exp \left(-c_{i} t\right)}{b_{1} \exp \left(\frac{-\pi^{2} t}{\mu \sigma d^{2}}\right)+\sum_{i=2}^{\infty} b_{i} \exp \left(-c_{i} t\right)} .
$$

It can be clearly seen that if $t$ goes to infinity, the relationship between the thickness of the material and the decay pickup voltage of the PEC sensor placed on that material can be approximately specified by

$$
\left|\frac{\mathrm{d} \ln [V(t)]}{\mathrm{d} t}\right| \approx \frac{\pi^{2}}{\mu \sigma d^{2}} .
$$

Given the material properties $\mu$ and $\sigma$, the material thickness can be mathematically computed via the gradient of $\ln [V(t)]$. Though the $\ln [V(t)]$ is also a curve, [9] demonstrates that the gradient obtained by linearisation of $\ln [V(t)]$ could be approximately employed in reality. In this work, it is proposed to find the gradient on the most linear part of the curve $\ln [V(t)]$. In equivalent words, the ALSFL of $\ln [V(t)]$ is searched; and the gradient of the established fitting line is utilized in computation of the material thickness. An approach 
of computing the ALSFL of a curve will be discussed in the next section.

\section{SignAL INTERPRETATION}

The section delineates a new and efficient approach of interpreting a decay curve signal collected by the PEC sensor as a thickness of the cast iron material. The method can be easily extended to other PEC signals applied for any other materials. The Savitzky-Golay filter is firstly introduced and then employed in the proposed algorithm to find a "preliminary" thickness.

\section{A. Savitzky-Golay filter}

Savitzky-Golay filter is also known as a digital smoothing filter [13]. The premise behind this filtering technique is to separate digitized samples into windows of data. In other words, after digitizing the continuous signal into equally spaced samples, the successive data windows are formed from the adjacent samples. In each data window, a fixed degree polynomial is fitted based on the sampled data. A dependent value of the polynomial function at the center of the window is a representative of the window data and is considered as a filtered (or smoothed) output value of the Savitzky-Golay filter in the corresponding window. The output values of the filter obtained in those windows are basically the smoothed signals. This makes the Savitzky-Golay filter more advantageous than other filters like standard average filter, where it does not significantly distort the original. It is assumed that there are $N$ samples from a signal, and $x(i)$ is one of the samples, $i=1, \cdots, N$. If cardinality of a window is $M$, from the samples $x\left(-\frac{M-1}{2}\right), \cdots, x\left(\frac{M-1}{2}\right)$, it can be obtained a corresponding polynomial vector. Now, it is supposed that if the input samples are the unit impulse $x(i)=\delta(i)$, their corresponding polynomial vector on each window is defined by $C\left(-\frac{M-1}{2}\right), \cdots, C\left(\frac{M-1}{2}\right)$. Here $C(i)$ is called a convolution coefficient. Therefore, the smoothed output value $y(i)$, corresponding to $x(i)$, of the Savitzky-Golay filter is specifically convoluted as follows.

$$
y(i)=\sum_{j=-\frac{M-1}{2}}^{\frac{M-1}{2}} C(j) x(i-j)
$$

In this work, the Savitzky-Golay filter is employed to smooth the raw signals. The results are then utilized to compute the "preliminary" thickness.

\section{B. Interpretation Algorithm}

In Section II-B, it is derived the closed-form solution of interpreting the thickness of assessed materials based on characteristics of the decay curve signals induced by the pickup coil of the PEC sensor. As demonstrated by equation (4) in time domain, given the material properties such as magnetic permeability and electrical conductivity, square of the material thickness is in linear relation to derivative of logarithm of the measured signal as time goes to infinity. Though it is unrealistic to compute the derivations in infinity, $\overline{\text { Algorithm } 1 \text { Algorithm for interpreting cast iron thickness }}$ based on Pulsed Eddy current

Input: Sampled signal, $\mu, \sigma$, standard table, maximum length of fitting line $\left(m_{l}\right)$, stopping threshold.

Output: Thickness of cast iron under sensor footprint.

1: Compute logarithm of voltage of sampled signal

2: Implement the Savitzky-Golay filter on computed logarithm values

3: Compute sum of logarithm of voltages

4: Infer a "preliminary" result of the thickness based on the standard table to decide a starting point (time domain)

5: while length of line $<m_{l}$ do

6: $\quad$ while stopping point $<$ stopping threshold do

7: $\quad$ Find a fitting line based on the linear least square

8: $\quad$ Compute average of distances $\leftarrow$ sum of all distances from samples between the starting and stopping points to the fitting line

9: $\quad$ Increase length of line

10: $\quad$ stopping point $\leftarrow$ starting point + length of line

11: end while

12: Increase length of line

13: end while

14: Adaptive least square fitting line $\leftarrow \underset{\text { fitting lines }}{\operatorname{argmin}}$ (average of distances)

15: Compute gradient of the adaptive least square fitting line 16: Thickness $\leftarrow$ compute equation (4)

the equation (4) still approximately holds on a specific part of the logarithmic decay curve. As a result, it is proposed to compute approximate linearisation of that specific part of the logarithmic decay signal. In equivalent words, by finding an ALSFL of the logarithmic curve of the measured signal, the thickness of the material under the sensor footprint can be easily obtained by computing a gradient of the ALSFL. Algorithmically, the fitting line from a set of samples can be obtained by the use of Random Sample Consensus (RANSAC) [10], [14]. Nevertheless, this renowned approach is also timeconsuming in computation. In literature, before processing the decay pick-up coil voltage curve, noises accompanied on the measured signals are suppressed. For instance, [5] proposed to compute an average signal from 40 repetitive sensor readings for each corresponding material thickness measurement. However, the method is infeasible in reality in which it requires 40 PEC sensor readings for assessing one material thickness [7]. As a consequence, in this work, we propose to employ only one decay curve from one PEC sensor reading to interpret the material thickness underneath the PEC sensor footprint. At first, the Savitzky-Golay filter is proposed to be utilized on the logarithmic values of the voltage signals in order to smooth and then suppress the noises on the logarithmic decay curve. This filter enables the system not to have to collect multiple measurements at one footprint. Furthermore, the smoothed values of the SavitzkyGolay filter are utilized to compute a sum of logarithms of the 


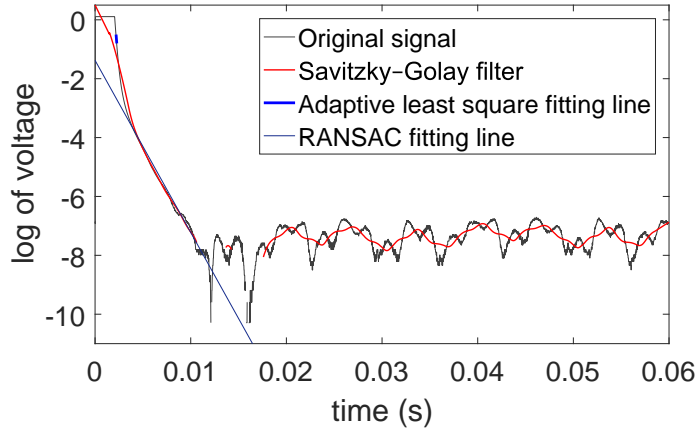

(a)

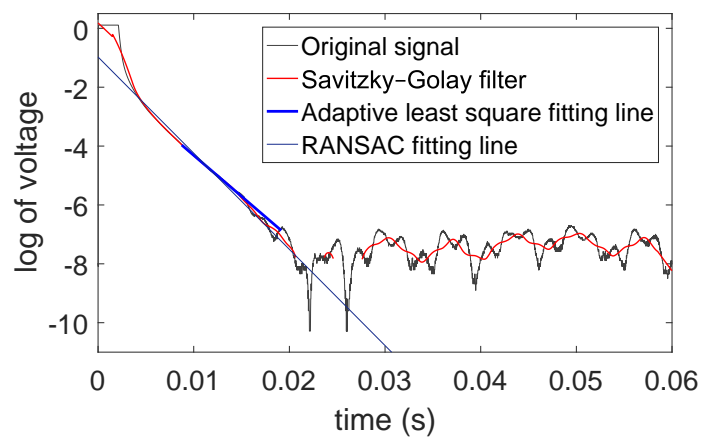

(c)

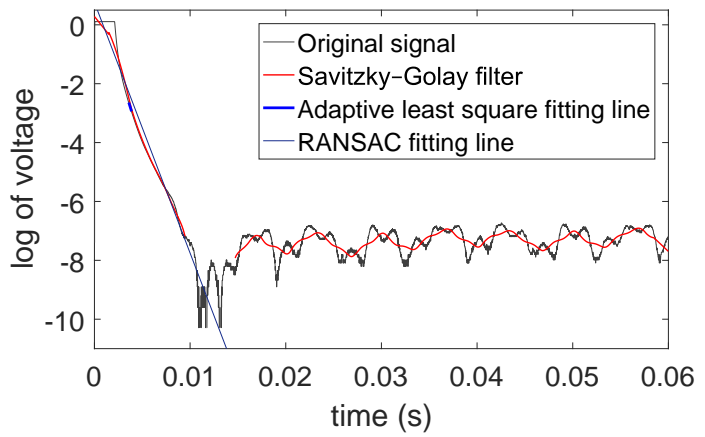

(b)

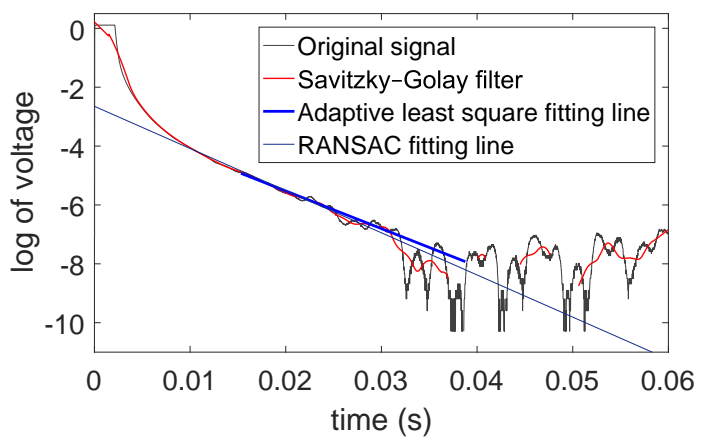

(d)

Fig. 2: The adaptive least square fitting lines of the logarithmic decay curves of the voltages collected by the PEC sensor: (a) $3 \mathrm{~mm}$, (b) $5 \mathrm{~mm}$, (c) $12.8 \mathrm{~mm}$ and (d) $20 \mathrm{~mm}$ cast iron thicknesses.

sensed samples, which is then used to infer a "preliminary" thickness as looking up a standard table. Note that each material thickness is represented by a specific decay curve. That is, sum of logarithms of voltage samples corresponding to one thickness is ideally unique. In this work, a standard table was created in the lab and specified by two columns of the thicknesses and their corresponding sum of logarithms of the voltage signal. Although the preliminary thickness obtained by looking up the standard table is not highly accurate due to noises in the realistic signal, it is employed to efficiently identify the specific part of the logarithmic decay curve, satisfying the equation (4). In other words, in the algorithm the preliminary thickness allows computing a starting point at which the program starts searching the ALSFL rather than searching from the beginning of the curve. In the next step of the algorithm, given a specific length of a line, fitting lines are calculated by using the linear least square method for successive sections on the decay curve during from the starting point to a stopping threshold, which is normally a very far end of the curve. The length of the fitting line is then increased; and other fitting lines are repeatedly obtained until the fitting line reaches a defined maximum length. Average of distances from all samples in each section to its corresponding fitting line is also computed. Ultimately, the fitting line whose average of distances is minimum is selected as the ALSFL.
The gradient of the ALSFL is easily quantified, which is substituted into the equation (4) to approximately compute the thickness. The algorithm of interpreting the thickness based on the PEC sensor is intuitively summarized in Algorithm 1. Fig. 2 illustrates examples of several decaying voltages induced by a real-life PEC pick-up coil placing on cast iron specimens with different thicknesses, which will be discussed in Section IV. While the original logarithmic decaying curves are highly noisy, the curves obtained by the Savitzky-Golay filter are quite smooth though they are not distorted from the original. More importantly, the adaptive least square fitting lines (ALSFLs) of these logarithmic voltage curves are also searched and demonstrated. It can be clearly seen that the length and position of the ALSFLs on the illustrative signals are comprehensively different. That is, the ALSFL, and then its corresponding gradient, is unique in each cast iron thickness. For instance, while the ALSFLs at low thicknesses in Figures $2 \mathrm{a}$ and $2 \mathrm{~b}$ are mostly located in the early first half of the curve, the ALSFL at a high thickness in Fig. $2 d$ is positioned at the far end of the signal. More interestingly, both the ALSFLs of the curves corresponding to $3 \mathrm{~mm}$ and $5 \mathrm{~mm}$ thicknesses, though very short, are located at the expected positions, which ultimately results in highly accurate estimations of the thicknesses. Other fitting lines obtained by the RANSAC algorithm are also demonstrated in Fig. 2. Visually, at high thicknesses 
the RANSAC fitting lines are approximately overlapped by those obtained by the proposed method, which does not hold at low thicknesses. Comparisons of their corresponding interpretation results will be discussed in Section IV.

\section{EXPERIMENTAL IMPLEMENTATION}

In this section, the configuration of the PEC sensing unit design, the testing implementation and results are presented to experimentally verify the proposed method.

\section{A. Experimental Design and Settings}

The structural diagram of the PEC sensor is schematically demonstrated in Fig. 3. A PEC sensor architecture consists of an exciter coil and a magnetic receiver, Ulapane [9] demonstrated that a non-receiver coil based design using magnetic sensors such as Hall-effect and magnetoresistive sensors has limited applicability; hence, in this work, a solenoid coil was employed as pick-up coil in the PEC sensor. In this design, the PEC sensor coupled coils are not direct in contact with the material. There is a insulation layer of lifting the sensor off. In the current work, a PEC sensing unit was tested on the different calibration cast iron plates. The whole testing system is illustrated in Fig. 4. For the purpose of evaluation, the parameters of the system were set as follows. Both excitation and pick-up coils, which are circularly wound by the copper wire with the American wire gauge index of 32 , are concentric. The driver circuit that supplies the pulses to the excitation coil was driven by a $20 \mathrm{~V}$ voltage source. The induced voltages from the pick-up coil are driven through the amplifier, where it has a gain of 2000. An opamp filter was also designed, whose cut-off frequency was set to $1 \mathrm{kHz}$. There was no inamp filter in this illustrative experiment. The decaying curve voltages are amplified to a range of $\pm 12.3 \mathrm{~V}$ before being digitized by an analog to digital converter of 16 bits. The testing was conducted on cast iron plates whose thicknesses are known and comprise 3.00, 5.00, 9.00, 12.80, 16.30, 18.00 and 20.00 $\mathrm{mm}$. As demonstrated in [9], the interpretation based on the PEC sensing is lowly influenced by the lift-off. That is, the gradients obtained on the different decaying curves induced at one location on the material, given correspondingly different lift-offs, are almost consistent.

\section{B. Data Acquisition}

The decaying curves induced by the pick-up coil of the PEC sensor, after being amplified by the amplifier and filtered, are transmitted to a personal computer (PC) via the Teensy USB development board, demonstrated in Fig. 4. In fact, the measured signals represented the cast iron thicknesses are sent to the PC through the USB port and read by a program written on the Matlab platform. The reading speed of the data acquisition program is about $60 \mathrm{~ms}$ per pulse cycle. Noticing that the cycle of the pulse in the excitation is $60 \mathrm{~ms}$. The decaying curve induced by the pick-up coil of the PEC sensor in each cycle corresponds to the falling edge of the pulse in the driver circuit. In other words, the reading speed of the Matlab based data acquisition program is able to on-line

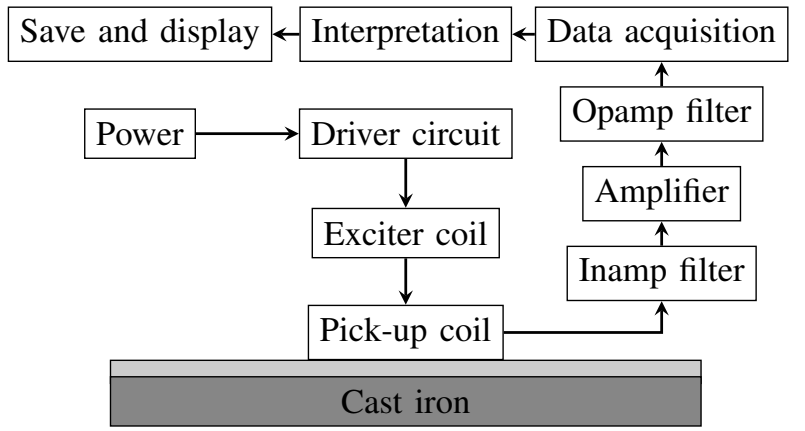

Fig. 3: Diagram of structure of the PEC sensing system for the cast iron.

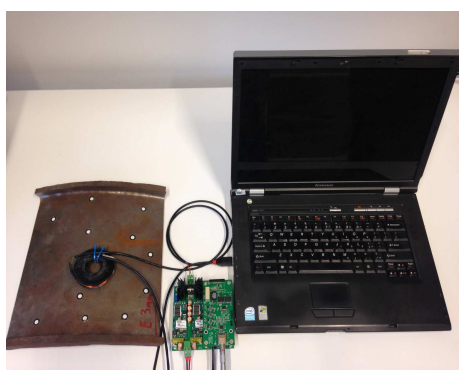

Fig. 4: Experimental implementation of the PEC sensing system.

collect the real-life measurements of the pick-up coil. In this experimental example, the sampling frequency was set to 100 $\mathrm{kHz}$. However, this frequency could be varied upon request. It is to be noted that the data acquisition program could also be written by any other languages such as $\mathrm{C}, \mathrm{C}++$ or Python.

\section{Experimental Results}

In the implementation, the concentric coils were sequentially placed on the top of some calibration cast iron plates whose thickness ranges are shown in Section IV-A. On each cast iron plate, the coils were located at a random positions; and 10 decaying curves of the induced voltages, corresponding to 10 pulses, were recorded at each location. After acquiring the data into the PC, the Algorithm 1 was utilized to compute the gradient of the ALSFL of the logarithmic decaying curve for each induced voltage signal. The gradient was then used to interpret the thickness of the cast iron where the pick-up coil was located, based on the equation (4) introduced in Section II-B. Note that there is only one average thickness presenting whole area under the pick-up coil footprint. Regarding the properties of the cast iron material being tested, high precision magnetization curves and electrical conductivity were obtained using a Quantum Design Physical Property Measurement System (PPMS-9T) [15]. In this implementation, $\mu=1.26 \times 10^{-4}$ $\mathrm{H} / \mathrm{m}$ and $\sigma=1.75 \times 10^{6} \mathrm{~S} / \mathrm{m}$. The interpreted thickness results are summarized in Table I, where the standard thicknesses 
TABLE I: INTERPRETATION OF CAST IRON THICKNESSES (mm) BASED ON THE PEC SENSOR'S MEASUREMENTS

\begin{tabular}{|c|c|c|c|c|c|c|c|c|c|c|}
\hline \multirow{3}{*}{$\begin{array}{l}\text { Standard } \\
\text { thicknesses }\end{array}$} & \multicolumn{10}{|c|}{ Interpreted thicknesses $(\mathrm{mm})$} \\
\hline & \multicolumn{5}{|c|}{ Proposed method } & \multicolumn{5}{|c|}{ RANSAC method } \\
\hline & Sig 1 & Sig 2 & Sig 3 & Avg of 5 & Avg of 10 & Sig 1 & Sig 2 & Sig 3 & Avg of 5 & Avg of 10 \\
\hline 20.00 & 19.10 & 19.03 & 18.92 & 19.04 & 19.00 & 17.66 & 17.50 & 17.83 & 17.63 & 17.74 \\
\hline 18.00 & 17.45 & 17.55 & 17.43 & 16.11 & 16.89 & 15.96 & 16.36 & 16.15 & 15.98 & 15.92 \\
\hline 16.30 & 16.20 & 16.09 & 15.21 & 15.51 & 15.64 & 14.56 & 14.49 & 14.62 & 14.61 & 14.25 \\
\hline 12.80 & 12.94 & 12.91 & 12.88 & 12.29 & 12.55 & 11.83 & 11.96 & 11.58 & 12.00 & 11.85 \\
\hline 9.00 & 9.24 & 9.22 & 9.16 & 9.30 & 9.34 & 8.58 & 8.45 & 8.49 & 8.58 & 8.52 \\
\hline 5.00 & 5.24 & 4.60 & 4.58 & 4.36 & 5.29 & 7.19 & 7.21 & 7.25 & 7.10 & 7.26 \\
\hline 3.00 & 3.40 & 3.30 & 3.31 & 3.41 & 3.42 & 7.75 & 7.57 & 7.30 & 8.00 & 7.58 \\
\hline
\end{tabular}

* Sig and Avg stand for Signal and Average, respectively.

of the calibration cast iron plates are also accompanied for the purpose of verifications. In this analysis, we run both the proposed algorithm and the RANSAC method to estimate the thicknesses from the decaying pick-up coil voltages. The $2^{\text {nd }}$ to $4^{\text {th }}$ and $7^{\text {th }}$ to $9^{\text {th }}$ columns of Table I present the resulting thicknesses of the tested plates by interpreting 3 random individual measurements out of 10 collected signals for each plate. However, the $5^{\text {th }}$ to $6^{\text {th }}$ and $10^{\text {th }}$ to $11^{\text {th }}$ columns delineate the thickness results by analysing the average signals of first 5 measurements and all 10 measurements, respectively, on each location. Overall, the proposed algorithm outperforms the RANSAC method. More importantly, in the proposed method, the result of an individual signal is highly comparable to that of an average signal. It can be apparently seen that our approach also plays the interpretations very well at low thicknesses, which the RANSAC method cannot interpret any signals from thicknesses being smaller than $7 \mathrm{~mm}$. In the context of computing time, we run both algorithms on the Matlab R2016a platform installed in a PC of Intel Core i5$6500 \mathrm{CPU} @ 3.20 \mathrm{GHz}$. The running time of the proposed approach and the RANSAC method to interpret a decaying voltage signal as a material thickness are $8 \mathrm{~ms}$ and $1001 \mathrm{~ms}$, respectively.

\section{CONCLUSION}

A signal processing approach for interpreting the decaying voltages induced by the pick-up coil of the PEC sensor as the thickness of the cast iron material has been presented, which is highly usable in non-destructive testing applications. The PEC inspection technology has been introduced, resulting in a derivation of an analytical solution of computing the material thickness based on the gradient of logarithms of the decaying voltage curve. Approximately, the proposed method searches for an adaptive least square fitting line of the logarithmic decaying curve, whose gradient is utilized in the thickness computation. Moreover, since it requires one measurement for each thickness estimation, in terms of testing speed the proposed algorithm outperforms the existing methods whose computation for each thickness value is usually based on multiple measurements. Our algorithm particularly has outperformance of better interpretation at low thicknesses and faster running time than conventional methods. The proposed approach has also been verified in a real-life cast iron thickness assessment system, where interpretation results are promising.

\section{REFERENCES}

[1] J. V. Miro, J. Rajalingam, T. Vidal-calleja, F. Bruijn, T. Wood, D. Vitanage, N. Ulapane, B. Wijerathna, and D. Su, "A live test-bed for the advancement of condition assessment and failure prediction research on critical pipes," Water Asset Management International, vol. 10(2), pp. 03-08, 2014.

[2] D. Vitanage, J. Kodikara, and G. Allen, "Collaborative research on condition assessment and pipe failure prediction for critical water mains," Water Asset Management International, vol. 10, pp. 15-18, 2014.

[3] Z. Liu and Y. Kleiner, "State of the art review of inspection technologies for condition assessment of water pipes," Measurement, vol. 46(1), pp. $1-15,2013$.

[4] W. Cheng, "Pulsed eddy current testing of carbon steel pipes wall thinning through insulation and cladding," Journal of Nondestructive Evaluation, vol. 31(3), pp. 215-224, 2012.

[5] C. Huang, W. Xinjun, X. Zhiyuan, and Y. Kang, "Pulsed eddy current signal processing method for signal denoising in ferromagnetic plate testing," NDT\&E International, vol. 43, pp. 648-653, 2010.

[6] C. Huang, X. Wu, Z. Xu, and Y. Kang, "Ferromagnetic material pulsed eddy current testing signal modeling by equivalent multiple-coilcoupling approach," NDT\&E International, vol. 44, pp. 163-168, 2011.

[7] C. Huang and X. Wu, "An improved ferromagnetic material pulsed eddy current testing signal processing method based on numerical cumulative integration," NDT\&E International, vol. 69, pp. 35-39, 2015.

[8] S. Huang and S. Wang, New Technologies in Electromagnetic Nondestructive Testing. Springer Singapore, 2016.

[9] N. Ulapane, "Nondestructive evaluation of ferromagnetic critical water pipes using pulsed eddy current testing," Ph.D. dissertation, University of Technology Sydney, Australia, 2016.

[10] N. Ulapane, A. Alempijevic, T. Vidal-Calleja, J. V. Miro, J. Rudd, and M. Roubal, "Gaussian process for interpreting pulsed eddy current signals for ferromagnetic pipe profiling," in Proc. IEEE Conference on Industrial Electronics and Applications, Hangzhou, China, June 2014, pp. $1762-1767$.

[11] J. García-Martín, J. Gómez-Gil, and E. Vázquez-Sánchez, "Nondestructive techniques based on eddy current testing," Sensors, vol. 11 (3), pp. 2525-2565, 2011.

[12] X. Chen and Y. Lei, "Excitation current waveform for eddy current testing on the thickness of ferromagnetic plates," NDT \& E International, vol. 66, pp. 28-33, 2014.

[13] S. J. Orfanidis, Introduction to Signal Processing. Englewood Cliffs, NJ: Prentice-Hall, 1996.

[14] M. Fischler and R. C. Bolles, "Random sample consensus: A paradigm for model fitting with applications to image analysis and automated cartography," Communications of the ACM, vol. 24(6), pp. 381-395, 1981.

[15] "Physical property measurement system," AC Measurement System (ACMS) Option Users Manual, Quantum Design, Part Number 1084$100 \mathrm{C}-1$. 